\title{
A novel DSF-like signal from Burkholderia cenocepacia interferes with Candida albicans morphological transition
}

\author{
Calvin Boon ${ }^{1,3}$, Yinyue Deng ${ }^{1,2,3}$, Lian-Hui Wang ${ }^{1}$, Yawen $\mathrm{He}^{1}$, Jin-Ling $\mathrm{Xu}^{1}$, Yang $\mathrm{Fan}^{1}$, \\ Shen Q Pan ${ }^{2}$ and Lian-Hui Zhang ${ }^{1,2}$ \\ ${ }^{1}$ Institute of Molecular and Cell Biology, Singapore and ${ }^{2}$ Department of Biological Sciences, National \\ University of Singapore, Singapore
}

\begin{abstract}
In addition to producing lethal antibiotics, microorganisms may also use a new form of antagonistic mechanism in which signal molecules are exported to influence the gene expression and hence the ecological competence of their competitors. We report here the isolation and characterization of a novel signaling molecule, cis-2-dodecenoic acid (BDSF), from Burkholderia cenocepacia. BDSF is structurally similar to the diffusible signal factor (DSF) that is produced by the RpfF enzyme of Xanthomonas campestris. Deletion analysis demonstrated that Bcam0581, which encodes an RpfF homologue, was essential for BDSF production. The gene is highly conserved and widespread in the Burkholderia cepacia complex. Exogenous addition of BDSF restored the biofilm and extracellular polysaccharide production phenotypes of Xanthomonas campestris pv. campestris DSF-deficient mutants, highlighting its potential role in inter-species signaling. Further analyses showed that Candida albicans germ tube formation was strongly inhibited by either coculture with $B$. cenocepacia or by exogenous addition of physiological relevant levels of BDSF, whereas deletion of Bcam0581 abrogated the inhibitory ability of the bacterial pathogen. As B. cenocepacia and C. albicans are frequently encountered human pathogens, identification of the BDSF signal and its activity thus provides a new insight into the molecular grounds of their antagonistic interactions whose importance to microbial ecology and pathogenesis is now becoming evident.
\end{abstract}

The ISME Journal (2008) 2, 27-36; doi:10.1038/ISMEJ.2007.76; published online 29 November 2007

Subject Category: microbe-microbe and microbe-host interactions

Keywords: BDSF; DSF; quorum sensing; signal interference; antagonism

\section{Introduction}

Microbe-microbe interactions are ubiquitous in various natural ecosystems. Not surprisingly, various forms of symbiosis and antagonism have surfaced. It has been known for a long time that microorganisms may produce antibiotics to inhibit or stop the growth of their competitors to gain competitive advantages. Recently, evidence is accumulating for a new form of microbial antagonism, which was tentatively designated as signal interference (Zhang and Dong, 2004). This type of antagonism acts not by killing, but instead by influencing the signal-mediated gene expression of the competitors and thus tips the balance of interaction (Dong et al., 2004; Hogan et al., 2004). For keeping up their competitive advantages, many

Correspondence: L-H Zhang, Institute of Molecular and Cell Biology, 61 Biopolis Drive, Proteos, Singapore 138673, Singapore. E-mail: lianhui@imcb.a-star.edu.sg

${ }^{3}$ These authors have contributed equally to this work.

Received 2 July 2007; revised 27 August 2007; accepted 27 August 2007; published online 29 November 2007 microorganisms appear to produce, release and respond collectively to species-specific small signal molecules to coordinate a range of important activities, such as virulence factor production, antibiotics biosynthesis and biofilm formation. This cell-cell communication mechanism is commonly known as quorum sensing (Whitehead et al., 2001; Fuqua and Greenberg, 2002). Logically, microorganisms might also boost their competitive strength in ecosystems by interfering with the quorum-sensing signaling of their competitors (Zhang and Dong, 2004).

Candida albicans causes various forms of candiasis ranging from mucosal infections to serious systemic infections. Mutants defective in morphological transitions during infection are avirulent (Lo et al., 1997; Braun et al., 2000; Saville et al., 2003; Zheng et al., 2003). Therefore, this ability to switch between yeast and hyphal-form is an important aspect of its pathogenesis. Intriguingly, the chance of infection by $C$. albicans seems to be influenced by the presence of certain bacterial pathogens. While the fungal pathogen can be found in various groups of patients who have undergone 
treatments with broad-spectrum antibiotics, it is rarely found in individuals suffering from cystic fibrosis that are chronically infected with Pseudomonas aeruginosa and Burkholderia cepacia (Kerr, 1994). This putative antagonism seems to be at least partially explained by the recent finding that the long-chain quorum-sensing signal 3-oxo-C12HSL produced by $P$. aeruginosa at physiological relevant level was sufficient to inhibit $C$. albicans yeast-tohyphae transition (Hogan et al., 2004). However, C8HSL, the major quorum-sensing signal produced by B. cepacia (Lewenza et al., 1999; Riedel et al., 2001), was not able to suppress the fungal filamentation (Hogan et al., 2004).

Burkholderia cepacia complex (BCC) has emerged as a major opportunist pathogen for immunocompromised individuals in particular the patients with cystic fibrosis and chronic granulomatous disease (Isles et al., 1984; Goldmann and Klinger, 1986; Mahenthiralingam et al., 2005). The Bcc consist of at least nine species that are phenotypically similar but genetically distinct, and all the species are capable of causing infections (Coenye et al., 2001; Mahenthiralingam et al., 2005). Among them, Burkholderia cenocepacia constitutes the majority of the transmissible and epidemic strains and is highly virulent (Mahenthiralingam et al., 2001, 2002). In this study, we report the identification and characterization of a novel signaling molecule designated as BDSF from B. cenocepacia. BDSF is a structural homologue of diffusible signal factor (DSF), which is the quorum-sensing signal produced by the plant bacterial pathogen Xanthomonas campestris pv. campestris $(X c c)$. In addition, we demonstrate that the ORF Bcam0581 is essential for BDSF production and can genetically substitute for the DSF synthase gene $r p f F$ in Xcc. Furthermore, we show that the dimorphic transition of $C$. albicans was inhibited by either exogenous addition of BDSF or coculturing with $B$. cenocepacia.

\section{Materials and methods}

Bacteria strains and growth conditions

B. cenocepacia J2315, a cystic fibrosis clinical isolate, was obtained from the American Type Culture Collection, Manassas, VA, USA. It is the representative strain of the highly transmissible ET12 clone (Govan et al., 1993). X. campestris pv. campestris strain 8004 and its $r p f F$ deletion mutant $8004 \mathrm{dF}$ were described previously (Wang et al., 2004; He et al., 2006b). XCC strains were maintained at $30^{\circ} \mathrm{C}$ in YEB medium (Zhang et al., 2002), while Escherichia coli and B. cenocepacia strains were grown in Luria-Bertani (LB) broth at $37^{\circ} \mathrm{C}$. The following antibiotics were supplemented when necessary: rifampicin, $50 \mu \mathrm{g} \mathrm{ml}^{-1}$; tetracycline, $10 \mu \mathrm{g} \mathrm{ml}^{-1}$ (XCC and E. coli) or $400 \mu \mathrm{g} \mathrm{m}^{-1}$ (B. cenocepacia); gentamycin, $100 \mu \mathrm{g} \mathrm{ml}^{-1}$; and trimethoprim, $300 \mu \mathrm{g} \mathrm{ml}^{-1}$. C. albicans SC5314 were grown either in GMM medium consisting of $6.7 \mathrm{~g}$ of Bacto yeast nitrogen base (Difco, Sparks, MD, USA) and $0.2 \%$ glucose or in minimum medium ( $\mathrm{pH} 7.2$ ). The latter consists of $\mathrm{K}_{2} \mathrm{PO}_{4}, 60 \mathrm{mM} ; \mathrm{KH}_{2} \mathrm{PO}_{4}, 30 \mathrm{mM}$; citrate, $20 \mathrm{mM}$; $\left(\mathrm{NH}_{4}\right)_{2} \mathrm{SO}_{4}, 15 \mathrm{mM} ; \mathrm{MgSO}_{4} \cdot 7 \mathrm{H}_{2} \mathrm{O}$, $0.8 \mathrm{mM} ; \mathrm{CaCl}_{2}, 90 \mu \mathrm{M} ; \mathrm{FeSO}_{4}, 30 \mu \mathrm{M} ; \mathrm{MnCl}_{2}, 15 \mu \mathrm{M}$; and Casamino acids, $0.5 \%$. DSF was added to the medium in a final concentration of $5 \mu \mathrm{M}$ unless otherwise indicated.

\section{Bioassay of BDSF}

The assay was performed as described previously using the biosensor strain FE58 (Wang et al., 2004). Briefly, 4-mm diameter wells were introduced on prepared bioassay plates and $20 \mu \mathrm{l}$ concentrated culture was added to each well. Alternatively, single colonies were spotted on bioassay plates. The plates were incubated at $30^{\circ} \mathrm{C}$ overnight. DSF activity is indicated by the presence of a blue halo around the well or colony.

\section{BDSF purification}

B. cenocepacia J2315 was grown in LB overnight with agitation at $37^{\circ} \mathrm{C}$. Seventy liters of culture supernatant was collected by centrifugation and extracted with equal volume of ethyl acetate. The crude extract (organic phase) was dried using a rotary evaporator and dissolved with methanol. The mixture was subjected to flash column chromatography using a silica gel column $(12 \times 150 \mathrm{~mm}$, Biotage Flash $12 \mathrm{M}$ cartridge) and eluted with ethyl acetate-hexane $(20: 80 \mathrm{v} / \mathrm{v})$. The active fractions were detected using the DSF bioassay and pooled. These were then concentrated, subjected to flash column chromatography again and eluted with ethyl acetate-hexane $(10: 90 \mathrm{v} / \mathrm{v})$. The purity of the collected active components was analyzed by highperformance liquid chromatography using a C18 reverse-phase column $(4.8 \times 250 \mathrm{~mm}$, Waters, Milford, MA, USA), eluted with methanol-water

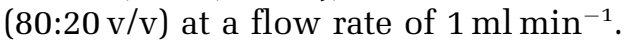

Structure analysis and synthesis of BDSF

${ }^{1} \mathrm{H},{ }^{13} \mathrm{C},{ }^{1} \mathrm{H}-{ }^{1} \mathrm{H}$ COSY, distortionless enhancement by polarization transfer, heteronuclear multiple bond coherence and heteronuclear multiple quantum coherence (HMQC) nuclear magnetic resonance (NMR) spectra in $\mathrm{CDCl}_{3}$ solution were obtained using a Bruker DRX400 spectrometer operating at $400 \mathrm{MHz}$ for ${ }^{1} \mathrm{H}$ or $100.5 \mathrm{MHz}$ for ${ }^{13} \mathrm{C}$. High-resolution electrospray ionization mass spectrometry was performed on a Finnigan/MAT MAT 95XL-T mass spectrometer. Conditions used were as stated before (Wang et al., 2004). BDSF was synthesized by Favorsky rearrangement of the corresponding 1,3dibromo-2-dodecanone as described previously (Wang et al., 2004). 
Construction of Bcam0581 in-frame deletion mutants B. cenocepacia J2315, a cystic fibrosis clinical isolate, was used as the parental strain to generate the Bcam0581 in-frame deletion mutants. The upstream and downstream regions flanking Bcam0581 were isolated using two PCR primer pairs, that is BCAM0581KO_LF (5'-ggatccctcgagatgcttgtcgaa), BCAM0581KO_LR ( $5^{\prime}$-aagcttggtatgtcctcgtgagatgtg); and BCAM0581KO_RF (5'-aagcttcgcacggtgtaatgcgac), BCA M0581KO_RR (5'-tctagaggatcc acgtatcgcgtgttctcgctg), respectively. This resulted in removal of $852 \mathrm{bp}$ of the 864 bp Bcam0581 coding sequence. To facilitate construction, BamHI and HindIII sites were included in the upstream fragment, whereas HindIII and XbaI sites were tagged to the downstream fragment. The PCR products were cleaved with respective enzymes and ligated to the suicide vector pEX18Tc (Hoang et al., 1998). The construct, verified by DNA sequencing, was introduced into B. cenocepacia J2315 by tri-parental mating with pRK2013 (Figurski and Helinski, 1979) as the mobilizing plasmid. The $B$. cenocepacia transconjugants were selected on LB agar plates containing tetracycline and gentamycin. Colonies harboring second crossover events were selected on LB agar containing 10\% sucrose. Bcam0581 deletion mutants ( $\triangle$ Bcam0581) were identified by colony PCR using the primer pair BCAM0581KO_LF and BCAM0581KO_RR described above.

\section{Complementation of strains $8004 d F$ and $\Delta B$ cam0581}

The coding region of Bcam0581 was amplified via PCR using primers pair BCAM0581-F (5'-ggatccatgcaactccaatcccatcc) and BCAM0581-R (5'-aagcttttacaccgtgcgcagctt). The product was digested with BamHI and HindIII and ligated separately to plasmid vectors pMSL7 (Lefebre and Valvano, 2002) and pLAFR3 at the same enzyme sites. The resultant construct was conjugated into $\triangle$ Bcam0581 and $8004 \mathrm{dF}$, respectively, by tri-parental mating. The transconjugants of $8004 \mathrm{dF}$ and $\triangle$ Bcam0581 were selected on YEB agar plates containing rifampicin and tetracycline and on LB agar plates supplemented with gentamycin and trimethoprim, respectively.

\section{Extracellular polysaccharide and biofilm analysis}

For quantification of extracellular polysaccharide (EPS) production, $10 \mathrm{ml}$ of overnight culture $\left(\mathrm{OD}_{600} \sim 2.5\right)$ were centrifuged at 12000 r.p.m. for 20 min. The collected supernatants were mixed with 2.5 volumes of absolute ethanol and the mixture was incubated at $4{ }^{\circ} \mathrm{C}$ for $30 \mathrm{~min}$. The precipitated EPS was isolated by centrifugation and dried overnight at $55{ }^{\circ} \mathrm{C}$ before determination of dry weights.

For analysis of biofilm formation, a single colony of each strain was inoculated and grown overnight at $37^{\circ} \mathrm{C}$ with agitation in $5 \mathrm{ml}$ of YEB medium. As a positive control, $5 \mu \mathrm{M}$ of DSF was added to Xcc
$8004 d F$ and methanol was added to the wild-type strain as solvent control. Multiple samples were obtained after overnight incubation and visualized with a phase contrast microscope (Olympus BX50). Imaging was performed using an Olympus DP70 digital camera.

Microscopic analysis and quantification of germ tube formation in $\mathrm{C}$. albicans

For testing the effect on $C$. albicans germ tube formation, BDSF and other compounds were diluted to appropriate concentrations in methanol. The overnight culture of $C$. albicans strain SC5314 grown in GMM medium were diluted 10-fold in fresh GMM medium containing the $3 \mathrm{kDa}$ fraction of fetal calf serum at a final concentration of $20 \%$. The $3 \mathrm{kDa}$ fraction was prepared by filtration of fetal calf serum through a membrane (Millipore, Billerica, MA, USA) with a $3 \mathrm{kDa}$ pore size and collection of the filtrates. This preparation was necessary as it drastically reduced the crude serum-induced aggregation but retained the potent germ tube induction capability, thereby allowing accurate quantification. The testing signal molecules were added separately to appropriate final concentrations as indicated and the cells were induced for $3 \mathrm{~h}$ at $37^{\circ} \mathrm{C}$. For growth experiments, overnight cultures $\left(\mathrm{OD}_{600} \approx 1.0\right)$ were diluted to $\mathrm{OD}_{600} \approx 0.06$ in GMM medium and cultured at $30^{\circ} \mathrm{C}$ with agitation (200 r.p.m.). Coculture experiments were performed in minimum medium using three $B$. cenocepacia strains, which displayed a similar growth rate. Fresh bacilli and yeast-form $C$. albicans were cultured together in the ratio of 20:1 with $E$. coli $\mathrm{DH} 5 \alpha$ as a negative control. The mix cultures were grown for $15 \mathrm{~h}$ at $30^{\circ} \mathrm{C}$ and then $3 \mathrm{~h}$ at $37^{\circ} \mathrm{C}$ for induction of germ tube formation. Quantification of germ tube formation was performed using a phase contrast microscope (Olympus BX50) by counting about 400 C. albicans cells per sample. Imaging was achieved using a Leica DMR Fluorescence microscope with $\times 100$ objective and a Hamamatsu digital camera interphased with METAMORPH software (Universal Imaging, Downingtown, PA, USA).

\section{Results}

Detection of DSF-like activity in B. cenocepacia In our preliminary screening, several environmental isolates, belonging to Burkholderia spp. based on $16 \mathrm{~S}$ rDNA analysis, were found capable of producing DSF-like signals when assayed using the DSF biosensor XCC strain FE58 (Wang et al., 2004). For further characterization, strain $B$. cenocepacia J2315, which is a clinical isolate with genome sequence available (http://www.sanger.ac.uk/cgi-bin/ blast/submitblast/b_cenocepacia), was obtained from ATCC. A single colony of strain J2315 was spotted on the bioassay plate containing the biosensor. A blue halo, which indicates the presence of DSF activity, 
was detected after incubation at $30^{\circ} \mathrm{C}$ overnight (Figure 1a), suggesting that B. cenocepacia J2315 may secrete a DSF-like molecule, which was tentatively designated as BDSF.

Bcam0581 is the rpfF homologue of B. cenocepacia J2315 essential for production of BDSF

In Xcc, a putative enoyl-CoA hydratase encoded by $r p f F$ is a key enzyme for DSF biosynthesis. Mutation of $r p f F$ abolishes DSF production and reduces the DSF-mediated virulence gene expression (Barber et al., 1997; Wang et al., 2004; He et al., 2006b). To identify the gene responsible for BDSF biosynthesis, the RpfF homologue was searched in the genome of B. cenocepacia J2315 by using the BLAST program (Altschul et al., 1990). The top hit is a peptide encoded by Bcam0581 showing a $37.2 \%$ identity with RpfF with an e-value of $1.8 \times 10^{-44}$. The gene is located on chromosome 2 and encodes a protein of $32 \mathrm{kDa}$. Interrogation of the B. cenocepacia J2315 genome sequence with the protein sequence of Bcam0581 did not reveal the presence of paralogues. Domain analysis using the pfam database version 22.0 (Finn et al., 2006) showed that Bcam0581 contained an enoyl-CoA hydratase domain similar to the RpfF enzyme of XcC (Figure 2a).
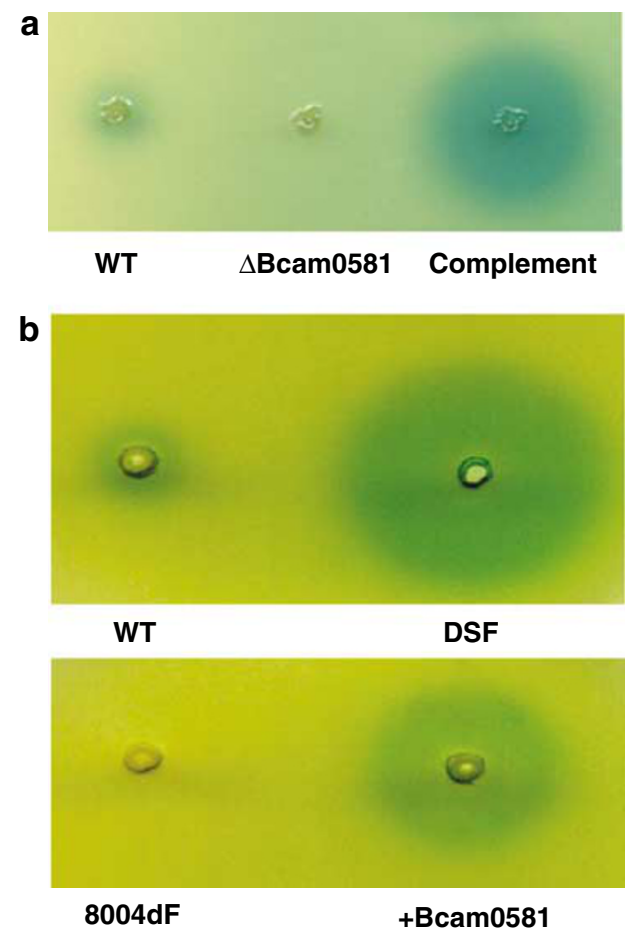

Figure 1 B. cenocepacia produced diffusible signal factor (DSF)like signals. (a) DSF bioassay with $B$. cenocepacia J2315 and derivatives. WT, wild-type $B$. cenocepacia J2315; complement, $\Delta$ Bcam0581(pMLS7-Bcam0581). (b) Expression of Bcam0581 in the DSF-deficient mutant $8004 d F$ of $X$. campestris pv. campestris $(X C C)$ restored DSF production. WT, wild-type XCC 8004; + Bcam0581, 8004dF(pLAF3-Bcam0581); DSF, $5 \mu \mathrm{l}$ of DSF $(5 \mu \mathrm{M})$. The presence of a blue halo around the site of inoculation indicates the presence of DSF-like activity.
The $r p f F$ of $X c c$ is located within the same locus as $r p f C$ and $r p f G$, which encode a DSF sensor and cognate response regulator, respectively. In contrast, no $r p f C$ or $r p f G$ homologue was found in the vicinity of Bcam0581 (Figure 2b), suggesting a different origin of evolution of the BDSF system in B. cenocepacia. The Bcam0581 gene appears to be a single transcriptional unit and is flanked by Bcam0582 and Bcam0580. The former encodes a $73 \mathrm{kDa}$ hypothetical protein and the latter a $73.3 \mathrm{kDa}$ protein with PAS, diguanylate cyclase (GGDEF) and a phosphodiesterase (EAL) domains. This domain structure is identical to that of PdeA from Xcc (Xc2324), which had been shown to regulate the synthesis of extracellular enzymes under oxygenlimited conditions (Ryan et al., 2007). In addition, protein blast analysis found that the peptide encoded by Bcam0580 was about 39\% identical to PdeA. Taken together, these observations indicate that Bcam0580 is a putative homologue of PdeA.

To verify the role of Bcam0581 in BDSF biosynthesis, the gene was deleted via homologous recombination. This resulted in a complete loss of BDSF production, and expression of the wild-type Bcam0581 gene in the deletion mutant resulted in overproduction of the signal (Figure 1a).

\section{Bcam0581 is a functional homologue of rpfF}

It was curious whether Bcam0581, which shows a moderate similarity to $r p f F$, could functionally replace the latter. For this purpose, the coding region of Bcam0581 was PCR-amplified and cloned under the control of the tac promoter in the expression vector pLAFR3. The resultant construct was conjugated into the $r p f F$ deletion mutant $8004 \mathrm{dF}$ (He et al., 2006b). As shown in Figure 1b, expression of Bcam0581 in the mutant restored DSF production. Furthermore, biofilm dispersal and EPS production were restored to wild-type levels in the strain $8004 \mathrm{dF}$ expressing Bcam0581 (Figure 3). Given that XCC is rather stringent in recognition and response to DSF-type signal molecules (Wang et al., 2004), these data suggest that Bcam0581 may produce the same DSF signal or a closely related structural analogue.

\section{BDSF purification and structural analysis}

The active component was collected from $70 \mathrm{l}$ of B. cenocepacia J2315 culture supernatants by ethyl acetate extraction and purified by flash column chromatography. About $7 \mathrm{mg}$ of BDSF was obtained from the combined active fractions after evaporation of the solvent. This was estimated at approximately $98 \%$ purity, based on the analysis by high-performance liquid chromatography.

The ${ }^{13} \mathrm{C}$ NMR spectrum showed that there were 12 peaks, which represent 12 carbons as stated below (Figure 4b). The nine ${ }^{13} \mathrm{C}$ peaks in the range of 14-32 p.p.m. were most likely from acyclic hydrocarbons. The ${ }^{1} \mathrm{H}-{ }^{13} \mathrm{C}$ correlated HMQC data 

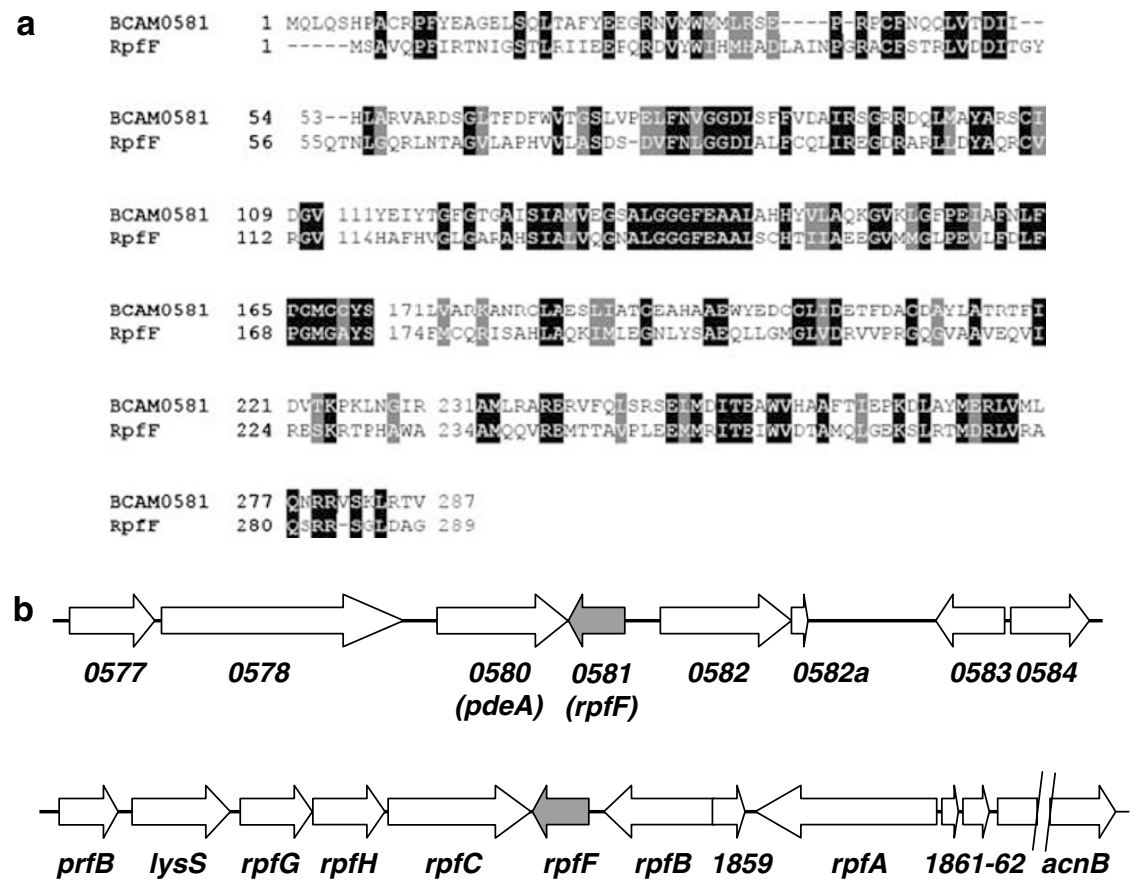

$1000 \mathrm{bp}$

Figure 2 Comparison of the peptide sequence and genomic organization of $r p f F$ and its homologue from B. cenocepacia. (a) Alignment of protein sequences of Bcam0581 with RpfF from Xcc. The black and gray shading indicates the identical and similar residues, respectively. (b) Genomic organization of the Bcam0581 region in B. cenocepacia J2315 (top) and that of rpfF region in XCC (bottom). The diffusible signal factor synthesis and response cluster ( $r p f A-r p f G)$ is flanked by peptide chain release factor (prfB); lysyl-tRNA synthase (lysS); aconitate hydratase $(a c n B)$ and two genes encoding hypothetical proteins (1861 and 1862). The region around the rpfF homologue Bcam0581 of B. cenocepacia consists of the genes encoding two transporters of the major facilitator superfamily (Bcam0577 and Bcam0584), a putative 5-oxoprolinase (Bcam0578), a PdeA homologue (Bcam0580), two hypothetical proteins (Bcam0582 and Bcam0582a) and an AraC-type transcription regulator (Bcam0583).

indicated that the peak at 14.08 p.p.m. was correlated with the three aliphatic protons at 0.88 p.p.m. (Figure 4a), probably representing a terminal $\mathrm{CH}_{3}$ group. The remaining eight ${ }^{13} \mathrm{C}$ peaks in the range of 14-32 p.p.m. represented eight $\mathrm{CH}_{2}$ groups based on HMQC and distortionless enhancement by polarization transfer spectra, suggesting the presence of an aliphatic chain. Among three carbon signals over 100 p.p.m., two at 118.91 and 153.43 p.p.m. were correlated with the two olefinic protons at 5.78 and 6.34 p.p.m., respectively, and were assigned to a double bond by HMQC spectrum. These two olefinic protons coupled to each other with a coupling constant of $11.5 \mathrm{~Hz}$, thus establishing the cis configuration of the double bond. A quaternary carbon peak at 171.34 p.p.m. should be assigned to a carbonyl, which conjugated to the double bond based on the ${ }^{1} \mathrm{H}^{13} \mathrm{C}$ correlated heteronuclear multiple bond coherence spectrum. Furthermore, high-resolution electrospray ionization mass spectrometry analysis of the purified BDSF revealed a molecular ion $(\mathrm{M}-\mathrm{H})^{-}$with an $\mathrm{m} / \mathrm{z}$ of 197.1532 , suggesting a molecular formula of $\mathrm{C}_{12} \mathrm{H}_{21} \mathrm{O}_{2}$ (197.1542). Taken together, these data indicate that BDSF is cis-2-dodecenoic acid (Figure 4c), a closely related structural analogue of DSF. In addition, we synthesized cis-2-dodecenoic acid and found that its
${ }^{1} \mathrm{H}$ and ${ }^{13} \mathrm{C}$ NMR spectra and biological activity were virtually indistinguishable from those of natural BDSF (data not shown).

BDSF inhibited the germ tube formation by C. albicans BDSF is structurally related to farnesol and highly similar to DSF. Both were shown to inhibit germ tube formation by C. albicans (Hogan et al., 2004; Wang et al., 2004). For determination of the potential inhibitory activity of BDSF against C. albicans, the signal was added to the fresh fungal yeast cells (Figure 5a). Farnesol and methanol were used as a positive and solvent control, respectively. After incubation at $37^{\circ} \mathrm{C}$ for $3 \mathrm{~h}$, more than $90 \%$ of C. albicans cells in solvent control formed germ tubes (Figure 5b), and farnesol at a final concentration of $5 \mu \mathrm{M}$ slightly reduced the length of germ tubes but seemed to have no effect on germination (Figure 5c). In contrast, BDSF at a final concentration of $5 \mu \mathrm{M}$ caused a marked reduction of germ tube germination and elongation (Figure $5 \mathrm{~d}$ ).

The above data suggest that BDSF is a highly potent inhibitor. Its activity was thus further compared quantitatively with several similar molecules, including DSF, 3OC12HSL and farnesol (Figures $5 \mathrm{e}$ and $\mathrm{f}$ ), using the same medium and 

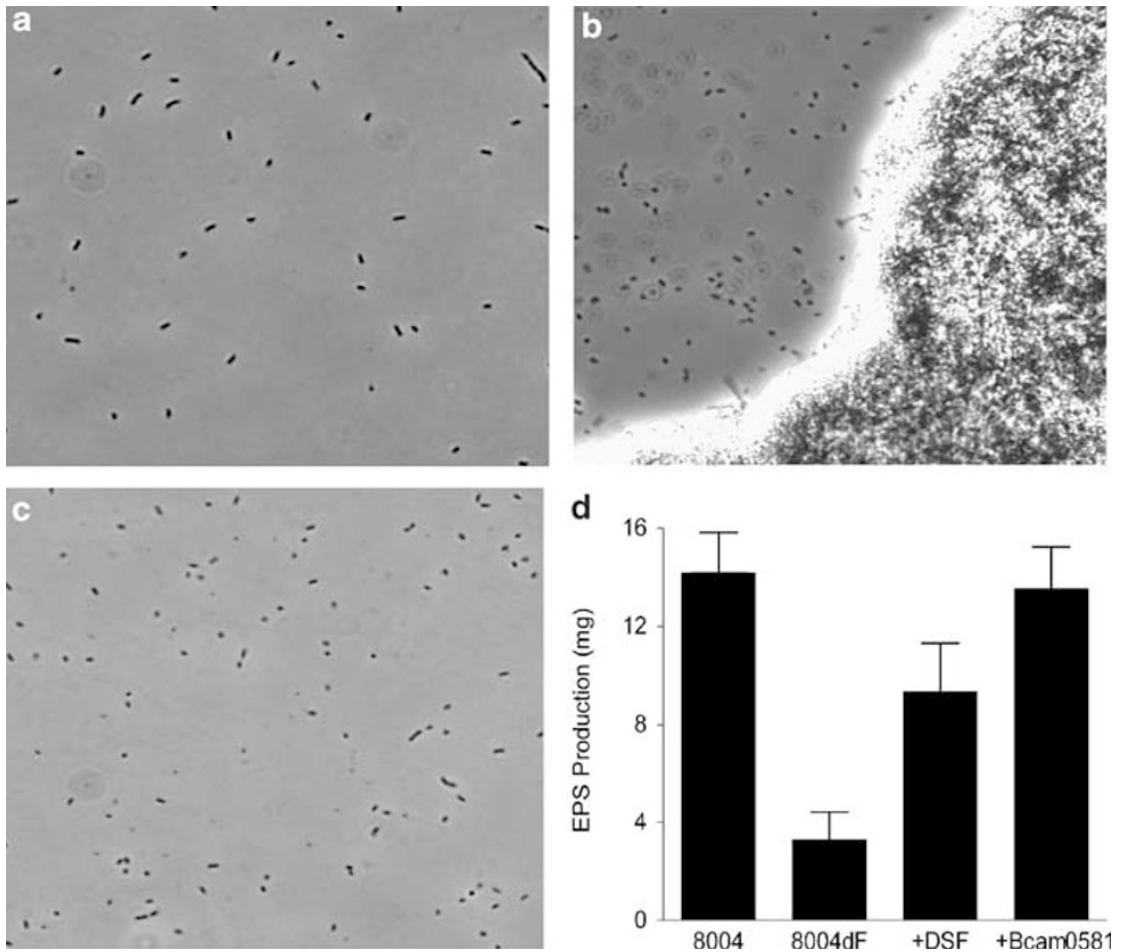

Figure 3 Complementation of the Xcc diffusible signal factor (DSF)-deficient mutant 8004dF by expression of the B. cenocepacia gene Bcam0581. (a) The cells of wild-type Xcc strain 8004 grew in planktonic (free-floating) form. (b) The $r p f F$ deletion mutant $8004 \mathrm{dF}$ derived from strain 8004 grew in biofilm (cell aggregates at the right-hand side of the photo) form. (c) Expression of Bcam0581 in trans in the mutant $8004 \mathrm{dF}$ dispersed the bacterial biofilm. (d) Extracellular polysaccharide (EPS) production levels. + DSF, exogenous addition of $5 \mu \mathrm{M}$ of DSF; + Bcam0581, mutant $8004 \mathrm{dF}$ expressing Bcam0581 in trans. Cells were observed under $\times 200$ magnification and the error bars show the standard deviations.

growth condition as described previously (Hogan et al., 2004). At a final concentration of $25 \mu \mathrm{M}$, none of them were able to inhibit yeast growth, but showed varied inhibitory effects on $C$. albicans germ tube formation with BDSF being the most effective, followed by DSF, farnesol and 3OC12HSL (Figure 5f). Consistent with the previous observation (Hogan et al., 2004), farnesol at a final concentration of $100 \mu \mathrm{M}$ caused about $45 \%$ reduction of germ tube formation (Figure 5f). It is also interesting to note that farnesol, 3OC12HSL and DSF at $100 \mu \mathrm{M}$ did no affect the yeast cell growth, but strikingly, BDSF at the same concentration showed a detrimental effect on yeast cell growth (Figure 5e). Further titration to a final concentration of $5 \mu \mathrm{M}, \mathrm{BDSF}$ and DSF resulted in about $60 \%$ and $12 \%$ reduction in germ tube formation, respectively, whereas farnesol and 3OC12HSL had no obvious effect (Figure 5f). Interestingly, even at $0.5 \mu \mathrm{M}$, BDSF outperformed its close structural analogue DSF at $5 \mu \mathrm{M}$ by reducing about $15 \%$ germ tube formation (Figure $5 \mathrm{f}$ ).

$\Delta$ Bcam0581 displays reduced ability to inhibit germ tube formation when cocultured with C. albicans To determine the ecological significance of BDSF, we grew $C$. albicans together with $B$. cenocepacia or its BDSF-deficient mutants as a mixed culture under hyphae-inducing conditions. Observation under the microscope showed that the presence of the wildtype B. cenocepacia J2315 significantly decreased the fungal morphological transition as a large percentage of $C$. albicans cells grew in yeast-form (Figure 6a). In contrast, majority of the fungal cells appeared as filaments when grown together with the BDSF-deficient mutant $\triangle B$ cam0581 (Figure 6b). Interestingly, the deletion mutant $\triangle B$ cam0581 formed heavy cell aggregates together with $C$. albicans hyphal cells (Figure 6b), whereas overexpression of Bcam0581 in the mutant resumed the planktonic phenotype and abolished the yeast-to-hyphal transition of C. albicans (Figure 6c). Quantitative analysis found that approximately $50 \%$ of the C. albicans cells counted were grown in hyphae-form when cocultured with $\Delta$ Bcam0581, which was close to the E. coli control mix culture where approximately $65 \%$ fungal cells were of hyphae-form. However, coculture of $C$. albicans with the wild-type B. cenocepacia J2315 decreased the hyphae cell level to about $20 \%$ (Figure 6d). The fungal hyphae cell level was further reduced down to only about $5 \%$ by the complementary strain that overproduced BDSF (Figures 1a and 6d), highlighting a BDSF dosage-dependent effect. 

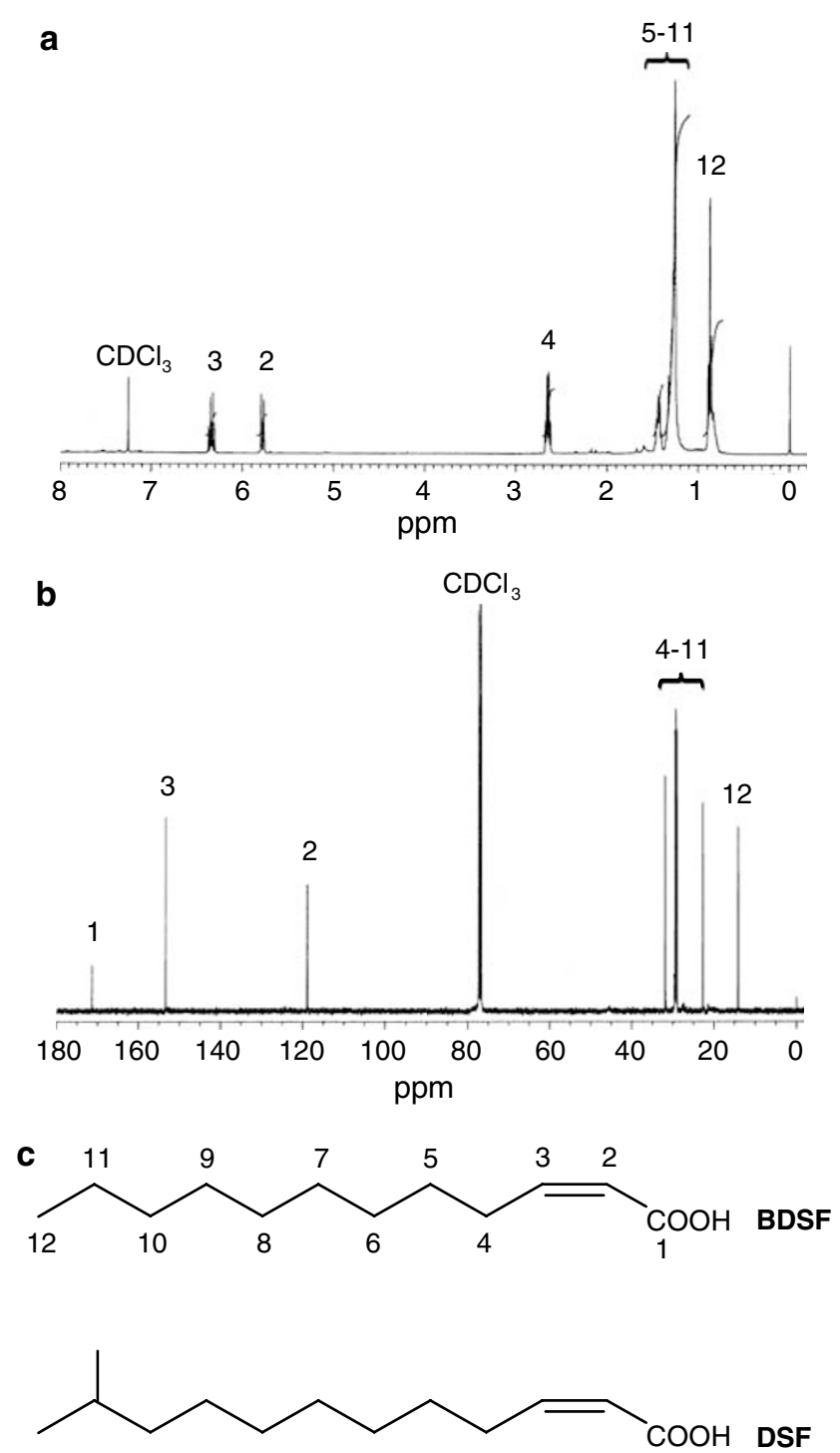

Figure 4 Purification and structural characterization of BDSF. (a) ${ }^{1} \mathrm{H}$ NMR spectral of BDSF. (b) ${ }^{13} \mathrm{C}$ NMR spectra of BDSF. (c) The predicted chemical structure of BDSF compared to diffusible signal factor. NMR, nuclear magnetic resonance.

\section{Discussion}

The results of this study show that the human opportunistic bacterial pathogen $B$. cenocepacia produces a potential cell-cell communication signal, which was structurally characterized as cis-2decenoic acid by mass spectrometry and NMR analysis (Figures 1 and 4). The structure of BDSF is similar to but not identical with the DSF (cis-11methyl-2-decenoic acid) signal produced by the plant bacterial pathogen Xcc (Wang et al., 2004). The only difference between the two molecules is at the C-11 position where BDSF lacks a methyl group. DSF is a well-characterized quorum-sensing signal that regulates a few hundreds genes encoding diverse biological functions through its signaling network comprising RpfC/RpfG two component system and a few transcriptional regulators (Ryan et al., 2006; He et al., 2006a, b, 2007). Xcc belongs to the $\gamma$-subdivision of proteobacteria, whereas $B$. cenocepacia is a member of the distantly related $\beta$-subdivision. Identification of BDSF from B. cenocepacia has strengthened the notion that DSF may represent a new class of conserved signals for bacterial cell-cell communications (Wang et al., 2004).

The BDSF biosynthesis by $B$. cenocepacia is encoded by the gene Bcam0581. This is supported by several lines of evidence. First, the peptide encoded by Bcam0581 shares about 37\% identity and a conserved enoyl-CoA hydratase domain with RpfF (Figure 2), the key enzyme known for DSF biosynthesis in XcC (Barber et al., 1997; Wang et al., 2004; He et al., 2006b). Second, expression of Bcam0581 in the rpfF deletion mutant of XcC restored the biofilm dispersal and EPS production to the wild-type level (Figure 3). Third, deletion of Bcam0581 in B. cenocepacia abolished the BDSF biosynthesis (Figure 1). Blast searches revealed the Bcam0581 homologues in the other five BCC genomovars with greater than $95 \%$ identity. They are B. cepacia sp 383 (genomovar I), B. multivorans (genomovar II), B. vietnamiensis (genomovar V), $B$. dolsa (genomovar VI) and B. ambifaria (genomovar VII). The presence of the Bcam0581 homologue in the remaining three $B C C$ genomovars is not clear as their genome sequences are not yet available. Thus, like CepIR, which is found in all strains of $B C C$ (Lutter et al., 2001), the BDSF signaling system may also be widely conserved in BCC.

Among the several reported bacterial and fungal signals, including the 3OC12HSL from $P$. aeruginosa, DSF produced by $X$. campestris and farnesol produced by $C$. albicans itself, BDSF showed the highest potency on inhibition of filament formation by $C$. albicans (Figure 5). The previous assays performed in different laboratories showed that DSF is more effective than 3OC12HSL for inhibition of germ tube formation (Hogan et al., 2004; Wang et al., 2004). This is consistent with our data that treatment of the fungal cells with about $25 \mu \mathrm{M}$ DSF or four times more 3OC12HSL resulted in about $45 \%$ reduction in germ tube formation (Figure $5 \mathrm{f}$ ). Highly significantly, BDSF was able to inhibit germ tube formation in approximately $70 \%$ of the cells at $5 \mu \mathrm{M}$. As DSF and BDSF differ only in the methyl group substitution at C-11 position, revealing such a structure-activity relationship is useful for further drug design and development. Furthermore, our data showed that at a high concentration $(100 \mu \mathrm{M})$, BDSF caused a complete growth inhibition of C. albicans, whereas DSF and other signals had no effect (Figure 5e). While the corresponding molecular mechanisms remain to be further investigated, these apparent dosage-dependent dual functions of BDSF on the fungal morphology and growth may present an exciting prospect for treatment of C. albicans infections. 

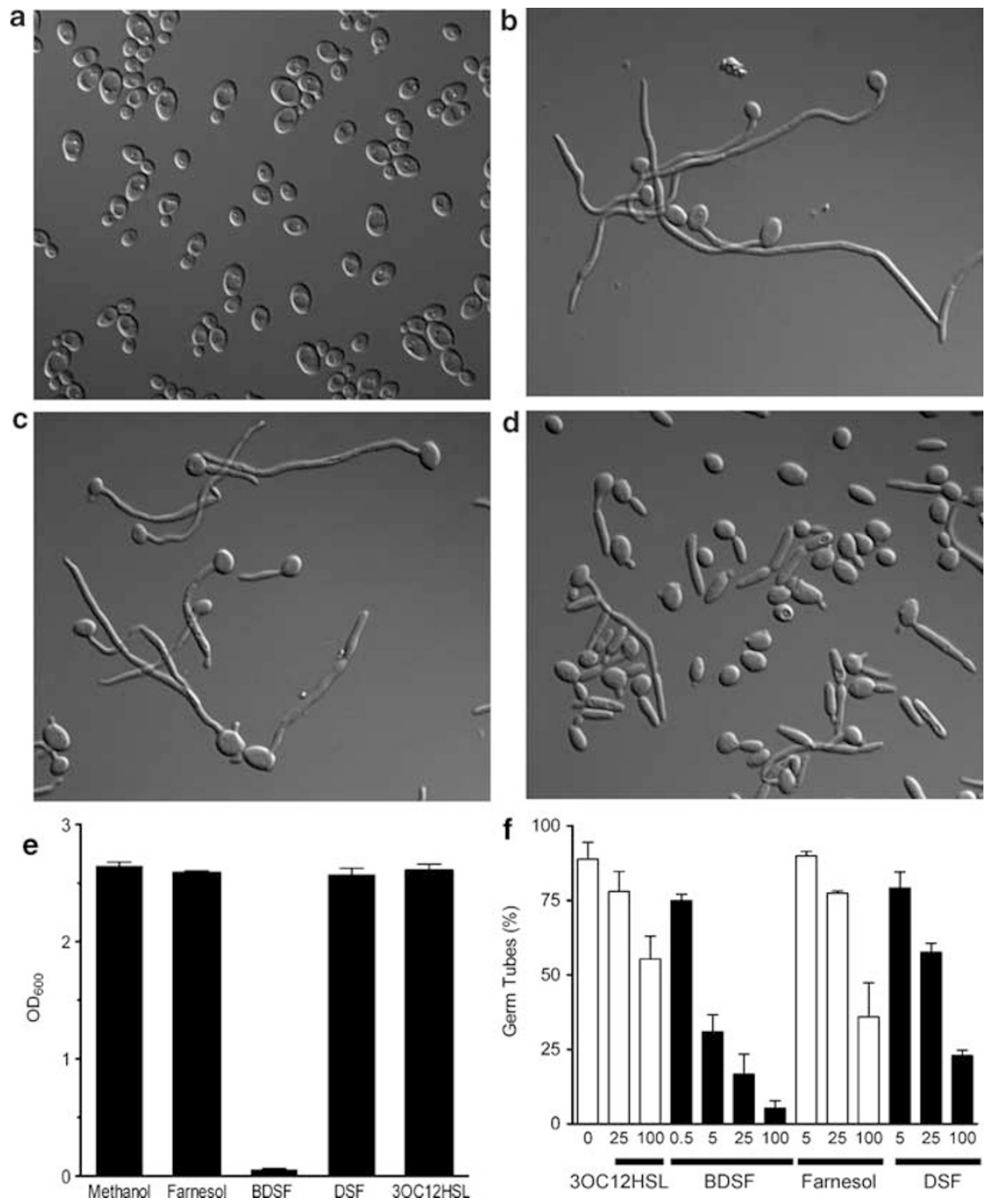

Figure 5 The effect of BDSF on C. albicans growth and germ tube formation. C. albicans cells were grown under non-induction conditions $\left(30^{\circ} \mathrm{C}\right)(\mathbf{a})$, or under induction conditions (serum extract, $\left.37^{\circ} \mathrm{C}\right)(\mathbf{b})$. In $(\mathbf{c}$ and $\mathbf{d})$, the cells were grown under the same condition as in (b) but treated with $5 \mu \mathrm{M}$ of farnesol and BDSF, respectively. The photos were taken $3 \mathrm{~h}$ after induction. (e) The effect of signal molecules on C. albicans yeast cell growth. The $\mathrm{OD}_{600}$ was determined $24 \mathrm{~h}$ after growth at $30^{\circ} \mathrm{C}$ with agitation. (f) Comparison of the inhibitory activity of BDSF and related signals on germ tube formation of C. albicans $3 \mathrm{~h}$ after induction. The experiment was performed twice, and each time at least 400 cells were counted per treatment. The error bars show the standard deviations.

The yield of $7 \mathrm{mg}$ of pure BDSF isolated from $70 \mathrm{l}$ of cultures translates to a minimum of $0.5 \mu \mathrm{M}$ present in overnight cultures of $B$. cenocepacia without taking into account the expected losses during the purification process. Significantly, even at this concentration, BDSF reduced the hyphal growth of C. albicans by about $15 \%$ (Figure $5 \mathrm{f}$ ), which suggests that the signal might play a role in cross-kingdom microbial competition in ecosystems. This speculation was demonstrated by the findings that deletion of the Bcam0581 gene significantly compromised the inhibitory effect of $B$. cenocepacia on the hyphal growth of $C$. albicans; and the mutant phenotype was rescued by complementation with the same gene (Figure 6). The ability to maintain an infection at a susceptible host site that is likely populated by several microbial species could be a combination of several factors. These may include the evolved survival mechanisms that are unique to ecological niche as well as signal interference and communication systems that can result in a competitive edge. There is increasing evidence that inter-genus and cross-kingdom communication is a widespread phenomenon (Zhang and Dong, 2004; Bassler and Losick, 2006). The ability of BDSF to phenotypically influence two organisms of different evolutionary lineage underscores its potential as a cross-kingdom and inter-genus signal, which may have a significant impact on the ability of Bcc to establish and maintain an infection in the host.

This work has uncovered a new DSF-like signal from bacterial pathogen B. cenocepacia and 

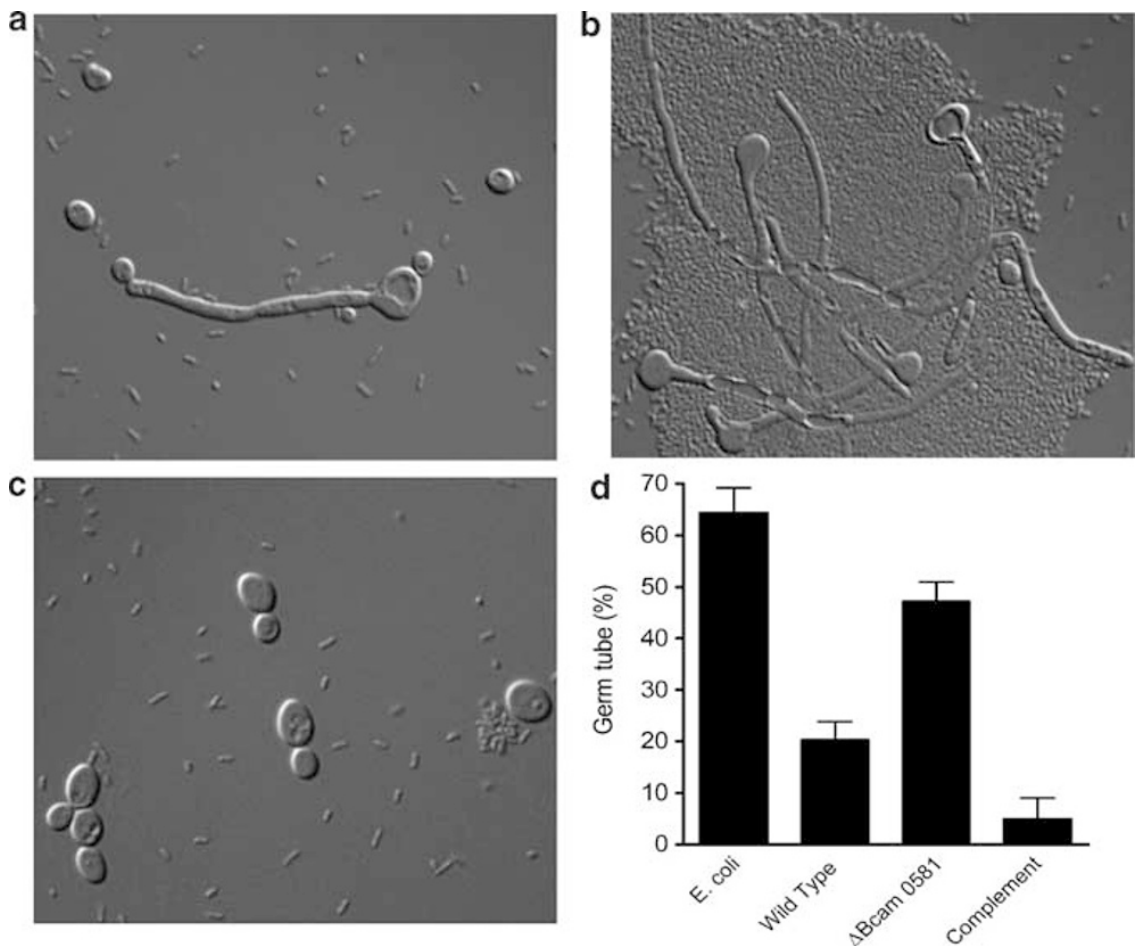

Figure 6 The inhibitory effect of $B$. cenocepacia on $C$. albicans germ tube formation was dependent on the BDSF synthase gene Bcam0581. C. albicans cells cocultured with (a) wild-type B. cenocepacia J2315, (b) BDSF-deficient mutant $\Delta B c a m 0581$ and (c) the complemented strain $\triangle B$ cam0581(pMLS7-Bcam0581). (d) The percentage of germ tube formation of C. albicans in the presence of different bacterial strains. E. coli strain DH5 $\alpha$ was included in the coculture experiment as a negative control. Experiment was repeated three times and each time at least 400 cells were counted per sample. The error bars show the standard deviations.

demonstrated its role in microbe-microbe interaction under in vitro conditions. Elucidation of the chemical structure and the gene encoding for synthesis of BDSF, which is highly conserved in $B C c$ complex, provides a new platform to explore potential genetic and signaling mechanisms that may modulate the physiology and virulence of these important bacterial pathogens. Furthermore, the finding that BDSF is a highly potent inhibitor on C. albicans hyphal growth raises intriguing questions on the molecular mechanism of signal interference and the potential role of this signal in competition between B. cenocepacia and C. albicans under in vivo conditions. Particular noteworthy is that the $B C C$ and $C$. albicans are frequent inhabitants of human and animals (Kerr, 1994; Hermann et al., 1999), identification of BDSF from B. cenocepacia thus underpins the potential ecological significance of DSF-like signals in bacteria-fungi interactions and competitions.

\section{Acknowledgements}

We are grateful to $\mathrm{M}$ Valvano for his generous gift of the plasmid pMLS7 and to Sanger Institute for providing us access to recent annotation of B. cenocepacia J2315 genome. We appreciate the advice provided by M Holden and E Mahenthiralingam on the ARTEMIS software and
Bcc strains, respectively. We thank the laboratory of Wang Yue for providing the SC5314 strain and technical advice in microscopy. This work was financially supported by the Agency of Science, Technology and Research (A*Star), Singapore.

\section{References}

Altschul SF, Gish W, Miller W, Myers EW, Lipman DJ. (1990). Basic local alignment search tool. J Mol Biol 215: $403-410$.

Barber CE, Tang JL, Feng JX, Pan MQ, Wilson TJ, Slater H et al. (1997). A novel regulatory system required for pathogenicity of Xanthomonas campestris is mediated by a small diffusible signal molecule. Mol Microbiol 24: 555-566.

Bassler BL, Losick R. (2006). Bacterially speaking. Cell 125: 237-246.

Braun BR, Head WS, Wang MX, Johnson AD. (2000). Identification and characterization of TUP1-regulated genes in Candida albicans. Genetics 156: 31-44.

Coenye T, Vandamme P, Govan JR, LiPuma JJ. (2001). Taxonomy and identification of the Burkholderia cepacia complex. J Clin Microbiol 39: 3427-3436.

Dong YH, Zhang XF, Xu JL, Zhang LH. (2004). Insecticidal Bacillus thuringiensis silences Erwinia carotovora virulence by a new form of microbial antagonismsignal interference. Appl Environ Microbiol 70: 954-960.

Figurski DH, Helinski DR. (1979). Replication of an origincontaining derivative of plasmid RK2 dependent on a 
plasmid function provided in trans. Proc Natl Acad Sci USA 76: 1648-1652.

Finn RD, Mistry J, Schuster-Bockler B, Griffiths-Jones S, Hollich V, Lassmann T et al. (2006). Pfam: clans, web tools and services. Nucleic Acids Res 34: 247-251.

Fuqua C, Greenberg EP. (2002). Listening in on bacteria: acyl-homoserine lactone signalling. Nat Rev Mol Cell Biol 3: 685-695.

Goldmann DA, Klinger JD. (1986). Pseudomonas cepacia: biology, mechanisms of virulence, epidemiology. $J$ Pediatr 108: 806-812.

Govan JR, Brown PH, Maddison J, Doherty CJ, Nelson JW, Dodd $\mathrm{M}$ et al. (1993). Evidence for transmission of Pseudomonas cepacia by social contact in cystic fibrosis. Lancet 342: 15-19.

He YW, Ng AY, Xu M, Lin K, Wang LH, Dong YH et al. (2007). Xanthomonas campestris cell-cell communication involves a putative nucleotide receptor protein Clp and a hierarchical signalling network. Mol Microbiol 64: 281-292.

He YW, Wang C, Zhou L, Song H, Dow JM, Zhang LH. (2006a). Dual signaling functions of the hybrid sensor kinase RpfC of Xanthomonas campestris involve either phosphorelay or receiver domain-protein interaction. J Biol Chem 281: 33414-33421.

He YW, Xu M, Lin K, Ng YJ, Wen CM, Wang LH et al. (2006b). Genome scale analysis of diffusible signal factor regulon in Xanthomonas campestris pv. campestris: identification of novel cell-cell communication-dependent genes and functions. Mol Microbiol 59: 610-622.

Hermann C, Hermann J, Munzelb U, Ruchel R. (1999). Bacterial flora accompanying Candida yeasts in clinical specimens. Mycoses 42: 619-627.

Hoang TT, Karkhoff-Schweizer RR, Kutchma AJ, Schweizer HP. (1998). A broad-host-range Flp-FRT recombination system for site-specific excision of chromosomally-located DNA sequences: application for isolation of unmarked Pseudomonas aeruginosa mutants. Gene 212: 77-86.

Hogan DA, Vik A, Kolter R. (2004). A Pseudomonas aeruginosa quorum-sensing molecule influences Candida albicans morphology. Mol Microbiol 54: 1212-1223.

Isles A, Maclusky I, Corey M, Gold R, Prober C, Fleming P et al. (1984). Pseudomonas cepacia infection in cystic fibrosis: an emerging problem. J Pediatr 104: 206-210.

Kerr J. (1994). Inhibition of fungal growth by Pseudomonas aeruginosa and Pseudomonas cepacia isolated from patients with cystic fibrosis. J Infect 28: 305-310.

Lefebre MD, Valvano MA. (2002). Construction and evaluation of plasmid vectors optimized for constitutive and regulated gene expression in Burkholderia cepacia complex isolates. Appl Environ Microbiol 68: 5956-5964.

Lewenza S, Conway B, Greenberg EP, Sokol PA. (1999). Quorum sensing in Burkholderia cepacia: identifica- tion of the LuxRI homologs CepRI. I Bacteriol 181: 748-756.

Lo HJ, Kohler JR, DiDomenico B, Loebenberg D, Cacciapuoti A, Fink GR. (1997). Nonfilamentous C. albicans mutants are avirulent. Cell 90: 939-949.

Lutter E, Lewenza S, Dennis JJ, Visser MB, Sokol PA. (2001). Distribution of quorum-sensing genes in the Burkholderia cepacia complex. Infect Immun 69: 4661-4666.

Mahenthiralingam E, Baldwin A, Vandamme P. (2002). Burkholderia cepacia complex infection in patients with cystic fibrosis. J Med Microbiol 51: 533-538.

Mahenthiralingam E, Urban TA, Goldberg JB. (2005). The multifarious, multireplicon Burkholderia cepacia complex. Nat Rev Microbiol 3: 144-156.

Mahenthiralingam E, Vandamme P, Campbell ME, Henry DA, Gravelle AM, Wong LT et al. (2001). Infection with Burkholderia cepacia complex genomovars in patients with cystic fibrosis: virulent transmissible strains of genomovar III can replace Burkholderia multivorans. Clin Infect Dis 33: 1469-1475.

Riedel K, Hentzer M, Geisenberger O, Huber B, Steidle A, $\mathrm{Wu} \mathrm{H}$ et al. (2001). $\quad N$-acylhomoserine-lactonemediated communication between Pseudomonas aeruginosa and Burkholderia cepacia in mixed biofilms. Microbiology 147: 3249-3262.

Ryan RP, Fouhy Y, Lucey JF, Crossman LC, Spiro S, He YW et al. (2006). Cell-cell signaling in Xanthomonas campestris involves an HD-GYP domain protein that functions in cyclic di-GMP turnover. Proc Natl Acad Sci USA 103: 6712-6717.

Ryan RP, Fouhy Y, Lucey JF, Jiang BL, He YQ, Feng JX et al. (2007). Cyclic di-GMP signalling in the virulence and environmental adaptation of Xanthomonas campestris. Mol Microbiol 63: 429-442.

Saville SP, Lazzell AL, Monteagudo C, Lopez-Ribot JL. (2003). Engineered control of cell morphology in vivo reveals distinct roles for yeast and filamentous forms of Candida albicans during infection. Eukaryot Cell 2: 1053-1060.

Wang LH, He Y, Gao Y, Wu JE, Dong YH, He C et al. (2004). A bacterial cell-cell communication signal with cross-kingdom structural analogues. Mol Microbiol 51: 903-912.

Whitehead NA, Barnard AM, Slater H, Simpson NJ, Salmond GP. (2001). Quorum-sensing in Gram-negative bacteria. FEMS Microbiol Rev 25: 365-404.

Zhang HB, Wang LH, Zhang LH. (2002). Genetic control of quorum-sensing signal turnover in Agrobacterium tumefaciens. Proc Natl Acad Sci USA 99: 4638-4643.

Zhang LH, Dong YH. (2004). Quorum sensing and signal interference: diverse implications. Mol Microbiol 53: 1563-1571.

Zheng XD, Wang YM, Wang Y. (2003). CaSPA2 is important for polarity establishment and maintenance in Candida albicans. Mol Microbiol 49: 1391-1405. 